\title{
Major Histocompatibility Complex Molecules on Parenchymal Cells of the Target Organ Protect against Autoimmune Disease
}

\author{
Hui Shao, Henry J. Kaplan, Deming Sun \\ Department of Ophthalmology and Visual Sciences, Kentucky Lions Eye Center, \\ University of Louisville, Louisville, Ky., USA
}

\begin{abstract}
Parenchymal cells of the autoimmune organ may only express major histocompatibility complex (MHC) molecules during the disease process. In this paper, we hypothesize that the appearance of MHC molecules on parenchymal cells may augment the activation of invading autoreactive $\mathrm{T}$ cells and either exacerbate or suppress local inflammation. It is speculated that like many biological responses this is a two-edge sword - namely, the expression of modest levels of MHC molecules may inhibit the activation of invading T cells, whereas overexpression of these molecules may promote activation of autoimmune $\mathrm{T}$ cells, enhancing the inflammatory cascade, thus leading to tissue damage.
\end{abstract}

Copyright (C) 2007 S. Karger AG, Basel

Autoreactive T cells are found in healthy people [1] and non-immunized animals [2]; however, most people do not develop autoimmune disease and the induction of autoimmune disease in animals requires specific treatment regimens and the use of particular genetic strains. It appears that autoimmune reactions are normally suppressed and that autoimmune disease results from breakdown of this suppression. Thus, a complete understanding of the pathogenesis of autoimmune uveitis requires knowledge of the suppressive mechanisms that are normally operative, but fail during disease. Additionally, pathogenic $\mathrm{T}$ cells may have attributes that are lacking in their non-pathogenic counterparts. One such protective mechanism is the ability of parenchymal cells of the target organ to inhibit autoreactive $\mathrm{T}$ cells. Indeed, an 'intrinsic 
abnormality of the target organs' has been previously proposed to explain the mechanism(s) by which autoreactive $\mathrm{T}$ cells mediate diseases $[3,4]$.

Experimental autoimmune uveitis (EAU), an autoimmune disease induced in experimental animals [5-8], has been, for many years, a popular laboratory model of human uveitis and even, to some extent, other T cell-mediated autoimmune diseases. EAU can be induced either by immunization of susceptible strains of rodents with a defined autoantigen or by adoptive transfer of autoreactive antigen-specific $\mathrm{T}$ cells.

\section{Active Experimental Autoimmune Uveitis}

By definition, active EAU is initiated by injection of ocular antigen in an immunogenic form, usually as an emulsion in complete Freund's adjuvant. This elicits a peripheral immune response, and, in susceptible animals, ocular inflammation of the eye. Generally, symptoms of uveitis in the rat appear by the 8th-10th day following immunization, persist for a little over a week, then subside. Antigen is taken up and processed into smaller peptide fragments that become complexed to major histocompatibility complex (MHC) molecules expressed on the surface of antigen-presenting cells (APCs). For example, when interphotoreceptor retinoid-binding protein (IRBP) is used as the eliciting antigen in the C57BL/6 mouse, a large portion of the immune response is directed against the 20-mer peptide containing residues $1-20$ of the protein (GPTHLFQPSLVLDMAKVLLD) $[9,10]$.

\section{Adoptive Transfer of Experimental Autoimmune Uveitis}

Somewhat simpler than active EAU, this begins with the transfer of lymphocytes from already immunized donors to recipients [10,11]. Thus, the initial stages of immunization, including adjuvant effects and the activation of disease-causing $\mathrm{T}$ cell subsets, do not occur in the recipients. $\mathrm{T}$ cells prepared from the lymph nodes of animals undergoing active EAU are restimulated in culture and adoptively transferred to syngeneic animals in which they cause inflammation in the eye and consequent tissue damage. Disease onset is more rapid than in active EAU, beginning on day 4 in rats $[5,12]$ and days $8-10$ in mice $[10,11]$. Adoptive transfer of a few million newly activated syngeneic autoreactive $T$ cells to a naïve animal can readily induce disease, suggesting the pathogenic role of autoreactive $\mathrm{T}$ cells in disease. The mechanism by which an organspecific autoimmune disease can be adoptively transferred by a few million autoreactive $\mathrm{T}$ cells, of which only a fraction enters the autoimmune organ, 
remains unclear. It is hypothesized that the entry of the pathogenic $\mathrm{T}$ cells provokes $\mathrm{MHC}$ expression on parenchymal cells and release of chemoattractant factors, which, in turn, recruit inflammatory cells.

\section{Autoreactive T Cell Lines and Clones}

To characterize the mechanism by which autoreactive $\mathrm{T}$ cells initiate autoimmune disease and to determine the various structural and functional features that distinguish between subsets of autoreactive $T$ cells and other antigenspecific, non-pathogenic $\mathrm{T}$ cells, enriched $\mathrm{T}$ cell populations have been prepared to determine the requirements for $T$ cell activation [12] and the usage of the $T$ cell antigen receptor [13-15] and accessory molecules [16, 17], as well as to assess the various cell-interacting cytokines produced by these cells [18-20]. The need for the characterization of the structure and function of autoreactive $\mathrm{T}$ cells by isolating antigen-specific $\mathrm{T}$ cell lines and clones became especially apparent when it is realized that limiting dilution analysis indicates that the number of autoreactive $\mathrm{T}$ cells in immunized rodents or in humans suffering from multiple sclerosis rarely exceeds 1 in $10,000 \mathrm{~T}$ cells $[21,22]$ and that the overwhelming majority of activated $\mathrm{T}$ cells associated with disease development are nonspecifically expanded [23].

\section{Retinal Pigment Epithelium}

Retinal pigment epithelial (RPE) cells, situated at a crucial interface between the choroidal blood supply and the photoreceptor cell layer of the neural retina, contribute to the immune-privileged status of the eye as part of the blood-eye barrier[24] by secretion of immunosuppressive factors inside the eye [25-28] and by expression of Fas ligand on their cell surface [29-31]. RPE cells may also assist in the development of intraocular inflammation [27, $32-34]$ and can respond to a variety of inflammatory cytokines [32, 35] and produce a myriad of molecules that can induce inflammation. For example, RPE cells can produce cytokines, such as tumor necrosis factor- $\alpha$, interleukin15 [36], and nitric oxide [37], and express cell surface MHC molecules and costimulatory molecules [38, 39]. In addition, RPE cells also express a number of uveitogenic antigens, such as soluble retinal antigen and IRBP, and could therefore become targets for uveitogenic $\mathrm{T}$ cells, leading to autoimmune reactions in the eye. While there is strong evidence that RPE cells can express MHC II molecules after activation $[39,40]$, the role of these molecules in the eye remains unclear. 


\section{Expression of Major Histocompatibility Complex Molecules by Retinal Pigment Epithelium}

One of the seminal findings of immunology is that the recognition of $\mathrm{T}$ cells is strictly restricted by antigens encoded by the MHC [41]. Based on this dogma, it is believed that only those parenchymal cells in the autoimmune organ capable of expressing MHC molecules can directly interact with the invading autoreactive $\mathrm{T}$ cells.

Under physiological conditions, RPE do not express appreciable levels of MHC II molecules [42] but do so during disease [43] or when they are activated in vitro by pro-inflammatory cytokines, such as interferon- $\gamma$ [44-46]. In the eye, aberrant expression of MHC molecules on RPE cells is therefore believed to alter disease susceptibility [47]. These cells, which can be induced to express MHC molecules and thus support T cell activation [44] in the eye, could be likely candidates for auto-attack.

The questions arise why parenchymal cells of the autoimmune organ retain the ability to express MHC II molecules but only do so during disease, and whether the appearance of MHC molecules in the autoimmune organ augments the activation of invading autoreactive $\mathrm{T}$ cells, and thus, exacerbates disease or, alternatively, restricts the intensification of local inflammation. It is speculated that like many biological responses this is a two-edge sword; whereas the expression of modest levels of MHC II molecules inhibits the activation of invading $\mathrm{T}$ cells, overexpression of these molecules promotes activation.

Thus, in early studies researchers tried to find evidence to support their hypothesis that the expression of MHC II molecules might render RPE able to stimulate autoreactive T cells [44]. Our studies have shown that depending on their state of activation, RPE cells can either inhibit or activate IRBP-specific $\mathrm{T}$ cells. In contrast to peripheral APCs, which elicit full activation (proliferation and cytokine release) of autoreactive T cells, RPE cells elicit only partial activation (tumor necrosis factor- $\alpha$ and interferon- $\gamma$ production, but not proliferation) [44].

\section{Retinal Pigment Epithelial Cells Expressing Major Histocompatibility Complex II Partially Activate Autoreactive T Cells and Drive These T Cells into a Refractory Phase}

The mere presence of MHC II molecules and appropriate antigen is not sufficient to induce $\mathrm{T}$ cell activation, and the presence of co-stimulatory molecules on MHC II-expressing cells is crucial [48, 49]. MHC II-expressing cells 
that lack accessory molecules may not only fail to function effectively as APCs, but can also result in unresponsiveness of T cells [49]. Conceivably, too low a density of accessory molecules on RPE cells may result in inhibition of T cell activation. We are currently investigating this possibility.

The availability of MHC II molecules in the autoimmune organ may cause the invading $\mathrm{T}$ cell to be activated. However, $\mathrm{T}$ cell biology studies tell us that two biological features of $\mathrm{T}$ cells have a closer relationship with the pathogenic activity of the autoreactive $\mathrm{T}$ cell. Firstly, the $\mathrm{T}$ cell can be activated to various degrees [50-53]. So-called 'partial activation' means that the T cells are activated, but only some of the activation-related $\mathrm{T}$ cell functions are turned on. Given that the damaging effect of autoreactive $\mathrm{T}$ cells is more closely correlated to the degree of activation than the number of $\mathrm{T}$ cells, partially activated $\mathrm{T}$ cells may have only limited pathogenic activity, possibly because they produce a lower pathogenic amount of damaging factors and are less cytotoxic. Secondly, and more importantly, both fully and partially activated $\mathrm{T}$ cells can enter a refractory phase. $\mathrm{T}$ cells are cycling cells and, once activated, can only been re-activated after a lag period. For both rat and mouse $\mathrm{T}$ cells, the duration of this cycle is approximately 5-7 days. Thus, immediately after entry into the autoimmune organ or before severe inflammation has been initiated, the expression of MHC II molecules allows the parenchymal cell to interact with the invading $\mathrm{T}$ cells. This interaction renders the invading $\mathrm{T}$ cells partially activated and they then enter a refractory phase; as a result, when professional APCs arrive at the peak of the inflammation, the refractory $\mathrm{T}$ cells cannot be reactivated. In this sense, the ability to express MHC molecules gives the parenchymal cell a protective capability, restricting the intensity of inflammation. This assumption has been tested in in vitro assays. Thus, we have examined whether the interaction of $\mathrm{T}$ cells and RPE affects $\mathrm{T}$ cell responsiveness to subsequent antigenic challenge by first treating $T$ cells with autoantigen in the presence or absence of RPE, then assessing their response to professional APCs. The results showed that pretreatment of $\mathrm{T}$ cells with RPE greatly decreased the ability of the $\mathrm{T}$ cells to respond to subsequent antigenic challenge $[19,44]$.

This assumption is also supported by the results of an in vitro experiment comparing the antigen-presenting activity of interferon- $\gamma$-activated RPE with that of professional APCs. We have observed that although RPE can activate autoreactive $\mathrm{T}$ cells, they are only $5-10 \%$ as effective as professional APCs. More importantly, T cells exposed to RPE expressing maximal levels of MHC II molecules produce only part of their cytokine repertoire compared to the same $\mathrm{T}$ cells stimulated by professional APCs. These observations led to the conclusion that unlike professional APCs, MHC II-expressing activated RPE can evoke only some of the functional properties of a $\mathrm{T}$ cell population [44]. 
Tissue damage provoked by invasion of autoreactive $\mathrm{T}$ cells appears to involve cascading responses in which the generation of cytokines and the recruitment of inflammatory cells reciprocally stimulate each other. Clearly, regulatory mechanisms are needed to control the intensity of inflammation and avoid tissue damage. It is hypothesized that the entry of autoreactive $\mathrm{T}$ cells elicits the release of cytokines or chemokines which then cause massive infiltration of inflammatory cells. Among the infiltrating cells are tissue-damaging cells, such as natural killer (NK) cells and macrophages, and others with antigen-presenting activity, such as dendritic cells and macrophages, resulting in further activation of the invading autoreactive $\mathrm{T}$ cells, leading to augmented infiltration and a cascading response.

Our studies have shown that MHC II molecule expression by parenchymal cells of the autoimmune organ plays a regulatory role in autoreactive $\mathrm{T}$ cell activation, and thus, the formation of an inflammatory response and tissue damage. This is because the activation of autoreactive T cells by the parenchymal MHC IIexpressing cells is only 'partial' and the production of pro-inflammatory cytokines is lower than pathogenic levels. Furthermore, this pre-activation renders the invading $\mathrm{T}$ cell refractory when potent professional APCs become available during the later phases of inflammation. In short, the expression of MHC molecules by the parenchymal cell of the autoimmune organ induces the invading $\mathrm{T}$ cell to become anergic after producing limited amounts of pro-inflammatory cytokines. It is also likely that the expression of MHC class I molecules protects glial cells from NK cell cytotoxic effects, as MHC-negative target cells are more vulnerable to cytolysis by NK cells [54]. Indeed, studies have shown that among the cells infiltrating the target organ during inflammation, a significant proportion possess an NK-like phenotype and cytotoxic activity [55].

Thus, the expression of MHC class II molecules by the parenchymal cell of the autoimmune organ is probably more beneficial than detrimental to the host in terms of preventing the full activation and expansion of potentially pathogenic T cells. Nevertheless, the production of incremental amounts of cytokines by $\mathrm{T}$ cells that are partially activated may also facilitate disease progression.

\section{Reciprocal Interaction between Autoreactive T Cells and Parenchymal Cells of the Eye}

We have previously shown that autoreactive $\mathrm{T}$ cells vary greatly in diseaseinducing capacity $[56,57]$. Unfortunately, this is not always reflected by differences in the fine specificity of the cell response or the cytokine-producing pattern of the maximally activated $\mathrm{T}$ cells. Because of this, researchers are searching for other cellular and molecular features showing a better correlation 
with the pathogenic nature of the cells. For example, studies in our laboratory have shown that the degree to which an autoreactive $\mathrm{T}$ cell is activated and its pattern of cytokine production are not innate to the cell and are not solely determined by the type of $\mathrm{T}$ cell receptor ligand that induces $\mathrm{T}$ cell activation, as the source of the APCs and the dose of antigen available are also important [58]. Given that the major MHC-expressing cells, such as glial cells in the central nervous system and astrocytes and RPE cells in the eye, may differ from professional APCs in the periphery in terms of antigen processing or accessory molecule expression, it is of interest to know whether activation of autoreactive $\mathrm{T}$ cells inside the autoimmune organ differs from $\mathrm{T}$ cell activation in the periphery, particularly in the presence of suboptimal doses of antigen, assuming that optimal in vitro doses would not always be available in vivo. It is possible, for example, that autoreactive $\mathrm{T}$ cell subsets capable of responding to limited antigen doses may pose a greater threat in vivo than other $\mathrm{T}$ cells with the same antigenic specificity, but activated only by larger doses of antigen.

Autoreactive $\mathrm{T}$ cell subsets differ greatly in their ability to interact with parenchymal cells $[59,60]$. This finding appears to be consistent with the previous observation that not all IRBP-reactive $\mathrm{T}$ cells produce a similar degree of tissue damage in the eye [12]. It remains to be determined whether the ability of $\mathrm{T}$ cells to interact with parenchymal cells of the autoimmune organ correlates with their pathogenic activity and whether pathogenic $\mathrm{T}$ cells have an enhanced or decreased ability to interact with parenchymal cells of the organ.

\section{Conclusion}

Although the physiological role of MHC molecules on parenchymal cells of the autoimmune organ is still poorly understood, it seems implausible that this expression of MHC II molecules during the genesis of autoimmune disease favors the reactivation of the invading autoreactive $T$ cell. Since disease is always preceded by inflammation of the diseased organ, a reaction that recruits large numbers of peripheral APCs which may cause stronger activation of the invading autoimmune $\mathrm{T}$ cell, it is assumed that expression of limited amounts of MHC II molecules by parenchymal cells should render the invading autoreactive $\mathrm{T}$ cells unresponsive to the infiltrating APCs by promoting their entry into a refractory phase of the cell cycle.

By their ability to express variable amounts of MHC molecules, parenchymal cells of the autoimmune organ, such as astrocytes and RPE cells, have the ability to control the degree of $\mathrm{T}$ cell activation in the organ. Thus, $\mathrm{T}$ cells entering the autoimmune organ and in contact with cells expressing low levels of MHC class II molecules downregulate their $\mathrm{T}$ cell receptor and become 
minimally activated and hyporesponsive. These observations support the premise that the primary role of MHC II-expressing cells in the autoimmune organ is to diminish or block full $\mathrm{T}$ cell activation in the organ, and thereby, prevent the release of harmful cytokines. It is possible that, during massive $\mathrm{T}$ cell infiltration or infection, the local cells then express higher levels of MHC molecules, promoting greater $\mathrm{T}$ cell activation with the accompanying production of pro-inflammatory cytokines.

Circumstantial evidence indicates that interactions between autoreactive $\mathrm{T}$ cells and the parenchymal cells of the autoimmune organ are highly versatile. For example, only activated encephalitogenic $\mathrm{T}$ cells are able to penetrate the blood-brain barrier [61] and cause experimental autoimmune encephalomyelitis [62]. The levels of MHC antigens expressed on parenchymal cells determines not only the activation of invading autoreactive $T$ cells, but also the survival of these parenchymal cells faced with the cytolytic activity of autoaggressive $\mathrm{T}$ cells [62]. In addition, the availability of $\mathrm{T}$ cell-specific antigen is critical for the cell-cell interaction [62] and for the persistence of the invading $\mathrm{T}$ cell in the organ $[63,64]$. Further studies should provide a better understanding of the pathogenesis of autoimmune ocular disease.

\section{Acknowledgment}

This study was supported by grants EY014366 (D.S.), EY12974, EY14599 (H.S.) and R24 EY015636 from the National Institutes of Health and the Research to Prevent Blindness Endowment Fund, New York, N.Y., USA.

\section{References}

1 Burns J, Rosenzweig A, Zweiman B, et al: Isolation of myelin basic protein-reactive T-cell lines from normal human blood. Cell Immunol 1983;81:435-440.

2 Schlüsener HJ, Wekerle $\mathrm{H}$ : Autoaggressive T lymphocyte lines recognizing the encephalitogenic region of myelin basic protein: in vitro selection from unprimed rat $\mathrm{T}$ lymphocyte populations. J Immunol 1985;135:3128-3133.

3 Massa PT, ter Meulen V, Fontana A: Hyperinducibility of Ia antigen on astrocytes correlates with strain-specific susceptibility to experimental autoimmune encephalomyelitis. Proc Natl Acad Sci USA 1987;84:4219-4223.

4 Chung IY, Norris JG, Benveniste EN: Differential tumor necrosis factor $\alpha$ expression by astrocytes from experimental allergic encephalomyelitis-susceptible and -resistant rat strains. J Exp Med 1991;173:801-811.

5 Shao H, Sun SL, Kaplan HJ, et al: Characterization of rat CD8 + uveitogenic T cells specific for interphotoreceptor retinal-binding protein 1177-1191. J Immunol 2004;173:2849-2854.

6 Donoso LA, Merryman CF, Sery T, et al: Human interstitial retinoid binding protein: a potent uveitopathogenic agent for the induction of experimental autoimmune uveitis. J Immunol 1989;143: 79-83. 
7 Chan CC, Nussenblatt RB, Wiggert B, et al: Immunohistochemical analysis of experimental autoimmune uveoretinitis (EAU) induced by interphotoreceptor retinoid-binding protein (IRBP) in the rat. Immunol Invest 1987;16:63-74.

8 Caspi RR: Experimental autoimmune uveoretinitis - rat and mouse; in Cohen IR (ed): Animal Models for Autoimmune Diseases: A Guidebook. New York, Academic Press, 1994, p 57.

9 Silver PB, Rizzo LV, Chan CC, et al: Identification of a major pathogenic epitope in the human IRBP molecule recognized by mice of the H-2r haplotype. Invest Ophthalmol Vis Sci 1995;36: 946-954.

10 Shao H, Peng Y, Liao T, et al: A shared epitope of the interphotoreceptor retinoid-binding protein recognized by the CD4+ and CD8+ autoreactive T cells. J Immunol 2005;175:1851-1857.

11 Shao H, Liao T, Ke Y, et al: Severe chronic experimental autoimmune uveitis (EAU) of the C57BL/6 mouse induced by adoptive transfer of IRBP1-20-specific T cells. Exp Eye Res 2006;82:323-331.

12 Shao H, Song L, Sun SL, et al: Conversion of monophasic to recurrent autoimmune disease by autoreactive T cell subsets. J Immunol 2003;171:5624-5630.

13 Sun D, Hu X, Le J, et al: Characterization of brain-isolated rat encephalitogenic T cell lines. Eur J Immunol 1994;24:1359-1364.

14 Sun D, Le J, Coleclough C: Diverse T cell receptor $\beta$ chain usage by rat encephalitogenic T cells reactive to residues 68-88 of myelin basic protein. Eur J Immunol 1993;23:494-498.

15 Sun D, Gold DP, Smith L, et al: Characterization of rat encephalitogenic $\mathrm{T}$ cells bearing non- $\mathrm{V}_{\mathrm{b}} 8$ T cell receptors. Eur J Immunol 1992;22:591-594.

16 Shao H, Fu YX, Song L, et al: LTbR-Ig treatment blocks actively induced, but not adoptively transferred, uveitis in Lewis rats. Eur J Immunol 2003;33:1743.

17 Shao H, Fu Y, Liao T, et al: Anti-CD137 mAb treatment inhibits experimental autoimmune uveitis by limiting expansion and increasing apoptotic death of uveitogenic T cells. Invest Ophthalmol Vis Sci 2005;46:596-603.

18 Sun D, Hu X, Shah R, et al: Production of tumor necrosis factor- $\alpha$ as a result of glia-T-cell interaction correlates with the pathogenic activity of myelin basic protein-reactive $\mathrm{T}$ cells in experimental autoimmune encephalomyelitis. J Neurosci Res 1996;45:400-409.

19 Sun D, Coleclough C, Whitaker JN: Nonactivated astrocytes downregulate T cell receptor expression and reduce antigen-specific proliferation and cytokine production of myelin basic protein (MBP)-reactive T cells. J Neuroimmunol 1997;78:69-78.

20 Sun D, Hu XZ, Liu XH, et al: Expression of chemokine genes in rat glial cells: the effect of myelin basic protein-reactive encephalitogenic T cells. J Neurosci Res 1997;48:192-200.

21 Olsson T, Sun J, Hillert J, et al: Increased numbers of T cells recognizing multiple myelin basic protein epitopes in multiple sclerosis. Eur J Immunol 1992;22:1083-1087.

22 Sun D, Wilson DB, Cao L, et al: The role of regulatory T cells in Lewis rats resistant to EAE. J Neuroimmunol 1998;81:177-183.

23 Werdelin O, McCluskey RT: The nature and the specificity of mononuclear cells in experimental autoimmune inflammations and the mechanisms leading to their accumulation. J Exp Med 1971;133:1242-1263.

24 Zinn KM, Benjamin-Henkind JV: Anatomy of the human retinal pigment epithelium; in Zinn KM, Marmor MF (eds): The retinal pigment epithelium. Cambridge, Harvard University Press, 2002, pp 3-31.

25 Farrokh-Siar L, Rezai KA, Semnani RT, et al: Human fetal retinal pigment epithelium suppresses the activation of CD4 ${ }^{+}$and CD8 ${ }^{+}$T-cells. Graefes Arch Clin Exp Ophthalmol 1999;237:934-939.

26 Faure V, Courtois Y, Goureau O: Inhibition of inducible nitric oxide synthase expression by TNF $\alpha$ and $\beta$ in bovine retinal pigmented epithelial cells. J Biol Chem 1997;272:32169-32175.

27 Holtkamp GM, Kijlstra A, Peek R, et al: Retinal pigment epithelium-immune system interactions: cytokine production and cytokine-induced changes. Prog Retin Eye Res 2001;20:29-48.

28 Liversidge J, Forrester JV: Antigen processing and presentation in the eye: a review. Curr Eye Res 1992;11:49-58.

29 Griffith TS, Brunner T, Fletcher SM, et al: Fas ligand-induced apoptosis as a mechanism of immune privilege. Science 1995;270:1189-1192.

30 Griffith TS, Ferguson TA: The role of FasL-induced apoptosis in immune privilege. Immunol Today 1997;18:240-244. 
31 Jorgensen A, Wiencke AK, la Cour M, et al: Human retinal pigment epithelial cell-induced apoptosis in activated T cells. Invest Ophthalmol Vis Sci 1998;39:1590-1599.

32 Crane IJ, Kuppner MC, Mckillop-Smith S, et al: Cytokine regulation of RANTES production by human retinal pigment epithelial cells. Cell Immunol 1998;184:37-44.

33 Crane IJ, Wallace CA, Mckillop-Smith S, et al: Control of chemokine production at the bloodretina barrier. Immunology 2000;101:426-433.

34 Devine L, Lightman S, Greenwood J: Lymphocyte migration across the anterior and posterior blood-retinal barrier in vitro. Cell Immunol 1996;168:267-275.

35 Jaffe GJ, Van Le L, Valea F, et al: Expression of interleukin-1 alpha, interleukin-1 beta, and an interleukin-1 receptor antagonist in human retinal pigment epithelial cells. Exp Eye Res 1992;55:325-335.

36 Kumaki N, Anderson DM, Cosman D, et al: Expression of interleukin-15 and its receptor by human fetal retinal pigment epithelial cells. Curr Eye Res 1996;15:876-882.

37 Liversidge J, Grabowski P, Ralston S, et al: Rat retinal pigment epithelial cells express an inducible form of nitric oxide synthase and produce nitric oxide in response to inflammatory cytokines and activated T cells. Immunology 1994;83:404-409.

38 Hamel CP, Detrick B, Hooks JJ: Evaluation of Ia expression in rat ocular tissues following inoculation with interferon-gamma. Exp Eye Res 1990;50:173-182.

39 Osusky R, Dorio RJ, Arora YK, et al: MHC class II positive retinal pigment epithelial (RPE) cells can function as antigen-presenting cells for microbial superantigen. Ocul Immunol Inflamm 1997;5:43-50.

40 Percopo CM, Hooks JJ, Shinohara T, et al: Cytokine-mediated activation of a neuronal retinal resident cell provokes antigen presentation. J Immunol 1990;145:4101-4107.

41 Zinkernagel R, Doherty PC: H-2-compatibility requirement for T cell-mediated lysis of target cells infected with lymphocytic choriomeningitis virus: different cytotoxic $\mathrm{T}$ cell specificities are associated with structures from H-2K or H-2D. J Exp Med 1975;141:1427-1436.

42 Wong GMW, Bartlett PF, Clark-Lewis I, et al: Inducible expression of H-2 and Ia antigens on brain cells. Nature 1984;310:688-691.

43 Traugott U, Scheinberg LC, Raine CS: On the presence of Ia-positive endothelial cells and astrocytes in multiple sclerosis lesions and its relevance to antigen presentation. J Neuroimmunol 1985;8:1.

44 Sun D, Enzmann V, Lei S, et al: Retinal pigment epithelial cells activate uveitogenic T cells when they express high levels of MHC class II molecules, but inhibit T cell activation when they express restricted levels. J Neuroimmunol 2003;144:1-8.

45 Benson MT, Shepherd L, Cottam D, et al: The expression of class I major histocompatibility antigens by human retinal pigment epithelium in vitro. Graefes Arch Clin Exp Ophthalmol 1992;230:184-187.

46 Detrick B, Newsome DA, Percopo CM, et al: Class II antigen expression and gamma interferon modulation of monocytes and retinal pigment epithelial cells from patients with retinitis pigmentosa. Clin Immunol Immunopathol 1985;36:201-211.

47 de KY, Naud MC, Bellot J, et al: Differential tumor necrosis factor expression by resident retinal cells from experimental uveitis-susceptible and -resistant rat strains. J Neuroimmunol 1994;55: $1-9$.

48 Schwartz RH: Costimulation of T lymphocytes: the role of CD28, CTLA-4, and B7/BB1 in interleukin-2 production and immunotherapy. Cell 1992;71:1065-1068.

49 Jenkins MK, Schwartz R: Antigen presentation by chemically modified splenocytes induces antigen-specific T cell unresponsiveness in vitro and in vivo. J Exp Med 1987;165:302-310.

50 Cao WX, Tykodi SS, Esser MT, et al: Partial activation of CD8 ${ }^{+}$T cells by a self-derived peptide. Nature 1995;378:295-298.

51 Evavold BD, Allen PM: Separation of IL-4 production from Th cell proliferation by an altered T cell receptor ligand. Science 1991;252:1308-1310.

52 Sloan-Lancaster J, Shaw AS, Rothbard JB, et al: Partial T cell signaling: altered phospho-zeta and lack of zap70 recruitment in APL-induced T cell anergy. Cell 1994;79:913-922.

53 Sousa CRE, Levine EH, Germain RN: Partial signaling by CD8 ${ }^{+}$T cells in response to antagonist ligands. J Exp Med 1996;184:149-157. 
54 Orihuela M, Margulies DH, Yokoyama WM: The natural killer cell receptor Ly-49A recognizes a peptide-induced conformational determinant on its major histocompatibility complex class I ligand. Proc Natl Acad Sci USA 1996;93:11792-11797.

55 Shao H, Van Kaer L, Sun SL, et al: Infiltration of the inflamed eye by NKT cells in a rat model of experimental autoimmune uveitis. J Autoimmun 2003;21:37-45.

56 Sun D, Coleclough C, Hu X: Heterogeneity of rat encephalitogenic T cells elicited by variants of the MBP (68-88) peptide. Eur J Immunol 1995;25:1687-1692.

57 Sun D, Hu X, Shah R, et al: The pattern of cytokine gene expression induced in rat T cells specific for myelin basic protein depends on the type and quality of antigenic stimulus. Cell Immunol 1995; 166:1-8.

58 Sun D, Le J, Yang S, et al: Major role of antigen-presenting cells in the response of rat encephalitogenic T cells to myelin basic proteins. J Immunol 1993;151:111-118.

59 Tabi Z, McCombe PA, Pender MP: Apoptotic elimination of Vb8.2+ cells from the central nervous system during recovery from experimental autoimmune encephalomyelitis induced by the passive transfer of Vb8.2 $2^{+}$encephalitogenic T cells. Eur J Immunol 1994;24:2609-2617.

60 Pender M, McCombe PA, Yoong G, et al: Apoptosis of $\alpha \beta$ T lymphocytes in the nervous system in experimental autoimmune encephalomyelitis: its possible implications for recovery and acquired tolerance. J Autoimmun 1992;5:401-410.

61 Wekerle H, Linington C, Lassmann H, et al: Cellular immune reactivity within the CNS. Trends Neurosci 1986;9:271-277.

62 Sun D, Wekerle H: Ia-restricted encephalitogenic T lymphocytes mediating EAE lyse autoantigenpresenting astrocytes. Nature 1986;320:70-72.

63 Kawakami N, Odoardi F, Ziemssen T, et al: Autoimmune CD4+ T cell memory: lifelong persistence of encephalitogenic $\mathrm{T}$ cell clones in healthy immune repertoires. J Immunol 2005;175: 69-81.

64 Flugel A, Berkowicz T, Ritter T, et al: Migratory activity and functional changes of green fluorescent effector cells before and during experimental autoimmune encephalomyelitis. Immunity 2001;14:547-560.

Dr. Henry J. Kaplan

Department of Ophthalmology and Visual Science, University of Louisville

301 East Muhammad Ali Boulevard

Louisville, KY 40202 (USA)

Tel. +1 502852 5466, Fax +1 502852 4595, E-Mail hank.kaplan@louisville.edu 